\title{
The Collision between Lacquer Decoration Technology and Modern Bag Design
}

\author{
Xiao-hua Lin ${ }^{1 . *}$, Xiao-hong Yuan ${ }^{1}$, Wei-Yin ${ }^{1}$, Chao-wei Lin $^{2}$ \\ XiYuan Road 200\#,ShangJie Town, MingHou Country, the School of Fashion and Art Engineering, Minjiang University 1. \\ Fujian de Yun Polytron Technologies Inc2, FuZhou, FuJian,350108,China \\ Email:120540961@qq.com
}

\begin{abstract}
In modern bag design, we attach importance to both aesthetic and practicability, make it meet the personalization and production needs in artistic creation and the requirements of the form of craftwork, so we combine the traditional art technology of lacquer with modern bag design to fully show the material of the lacquer and the craftwork beauty by means of a richer artistic form, and bring new aspiration and expressive techniques to bag design.
\end{abstract}

\section{Keywords-Lacquer art; Bag design; Decoration; Collision}

\section{INTRODUCTION}

Bags, as an important part of clothing matching, not only accord with people's need for their practicability, are also becoming what the trendsetters use to show their own tastes by the bags' decorative effect, material and the characteristic of hand -custom made. Modern bags can be divided into two parts: first, the bag design which meets the practical need of daily life second, the bag design which meets the need of decoration. No matter what the bags are, we have higher demand on delicate workmanship, the particularity of decoration material and the color-matching.

\section{THE COMPROMISE OF LACQUER DECORATION TECHNOLOGY AND MODERN BAG DESIGN}

As a part of the traditional culture of China, lacquer decoration technology is still a kind of representative culture, proved by the practice for thousands of years. The mature technology and decoration effect have been shown since Han dynasty in China. In different age, we create different forms of expression. No matter from the daily necessities or the ornamental artistic works, we can enjoy the exquisite glamour of lacquer decoration technology and the uniqueness it brings to the products.

Leather, cotton and hemp, and nylon, etc, are normally used in modern bag design. Brand bags use more superior leather. But bags made of leather would easily be aging and have scratches in the process of use. However, proved by practice of long period, lacquer is able to protect the wrapped material very well, which brings new life to the leather bag design. It will greatly improve the bags' decoration function and artistry to combine the special material and technology utilizations, such as mother-of-pearl mosaic, gold -silver mosaic, bone-stone mosaic, eggshell mosaic, gold and silver foil, flash powder, plant and linen, etc, with decorative methods, like splicing, ribbon, cording, petit motif, metal fittings, beads, feathers and embroidery, etc, of traditional bag design in modern bag design. It is believed that the application of lacquer decoration technology in modern bag design will bring new trend again in bag design.

\section{APPLICATIONS OF LACQUER DECORATION TECHNOLOGY IN BAG DESIGN}

Designers generally use fabrics like leather, cotton and linen, nylon and so on in bag design. However, the leather in bags of leather fabric is easy to be aging and have scratches, etc, in using. And lacquer, proved by a long time practice, is able to protect the wrapped materials very well, which can bring more "permanent" vitality to bag design of leather fabrics. It will greatly improve the bags' decoration function and artistry to combine the special materials and technology utilizations, such as mother-of-pearl mosaic, gold -silver mosaic, bone-stone mosaic, eggshell mosaic, gold and silver foil, etc, with decorative methods, like splicing, lace, metal fittings, embroidery, etc, in bag design.

Designers of the world brand, GUCCI, wonderfully applied the materials like bamboo, flax, etc, in bag design because of the lack of leather during the time of fascist dictatorship, which made GUCCI survive through the hard times and create a unique characteristic for the brand. Nowadays, designers apply the lacquer decoration technology into bag design and it is believed to set off a new fad for bag design.

Lacquer as liquid lack of the directness of modeling and normally need model together with other materials, like fibre, leather, wood and metal. The materials normally used in bags are leather, fibre and metal, which are exactly the same with the materials of the needed medium when the lacquer is modeling. The modern bag design consists of these parts such as body, belting, handle, ornaments and hanging cards. The modeling of bags is shown by shape, space, colors and materials. Therefore, the opening locations, the decorations and the brand logos of bags are the important points in modern bag design.

Fund Project: Fuzhou Municipal Science and Technology Bureau project (2017-G-105). 


\section{A. The Application of Lacquer Decoration Technology in Bag Fittings}

The decoration effect of the handles, as the important parts of bags, could fatter the whole effect of the bags very well. For example, in Pic-1, the handle is wooden. We would use sandpaper to polish the handle slightly, paint yellow lacquer, stick on the mother-of-pearl when the lacquer is not completely dry and put it in the shade until it is dry. Later, we would paint green and white lacquer with gold power, use the sandpaper to sand down the handle until the colors of yellow, green and white appear in turn after it is completely dry in the shade again. At last, we would use dumpling flour with plant oil to push light until the mother-of-pearl texture appears. In Pic-2, we would first paint a layer of clear lacquer on the handle of the bag, stick on some eggshell to make the shape of flowers and put on some silver foil irregularly, and then paint red lacquer on the handle except the place of eggshells after the clear lacquer is completely dry. We would paint a layer of clear lacquer again after the red lacquer is dry. At last, we would push light after the second layer of clear lacquer is completely dry. This process will make the bag handle milder and more exquisite because of lacquer and polishing and make the bag handle glossier with the time goes by.

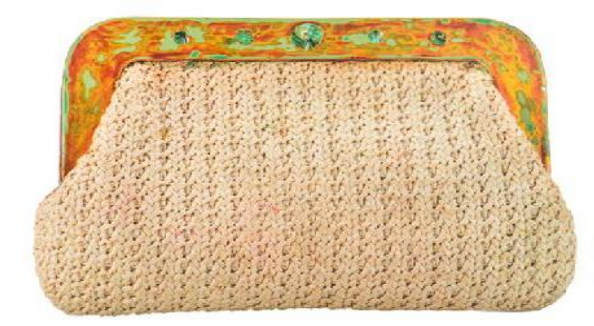

Fig. 1. Mother of pearl inlay method is applied to the packing bag

According to the bags' style design and the design pattern to match, we then use lacquer decoration technology in the bags' agrafes, which greatly improves the bags' artistry and their collection value. The agrafes are especially important in the simple design of the bags. For example, in Pic-3, we first paint lacquer on the agrafe, throw some sawdust on the lacquer and place it in the room of shade to dry, using the technology of mosaic. And then they would paint green and white lacquer and make it dry after the mother-of-pearl mosaic, and continue to paint yellow and white lacquer, and after it dries, paint blue color, then paint a layer of clear lacquer when the blue layer is dry and when the clear lay is about to dry, we would throw some gold flour and at last paint another layer of clear lacquer. We will polish the agrafe after it is completely dry. This is the way how an artistic agrafe comes into being.

\section{B. The Application of Lacquer Decoration Technology in the Bags Modeling}

The carrier of the traditional lacquer art is always the utensils with the value of practicality and decoration, while modern bags happen to accord with the practical value of utensils in lacquer art, which perfectly show the feature of "external appearance corresponding to inside" in which the "Lacquer" is as the external appearance and the "Utensil" is as the inside.

We generally emphasize practicality, simplification and convenience in traditional bags design, therefore, the common shapes of traditional bags are regular geometries, like the cuboid, the cube and the circular - arc. With the change of aesthetic taste and the abundance of fashion style, bags of various styles emerge in an endless stream. Bags, whose different kinds of innovative models are meeting people's need of seeking for new and difference, are the important part of dress collocation, the bags' appearance is one of the basic factors which can decide the bags design style. All the structure designs applied in the traditional bag design have many formularies and ductility manipulations. The relevant modeling features compromise to the factors, like equipments, materials and manufacturing technology and so on, to shape the manufacturing rules and process. But it is hard to make the innovative bag models really into the productions in the traditional designs of bags' modeling and structures. While the manufacturing methods of bodiless lacquerware in lacquer art can be well used in the bag design and make the demanded bag models: the leather used as the body calls "leather clothing" or "Weiyi". We will macerate it and model, and then produce various irregular artistry bag models based on different needs, and shape it after it dries, all of which will make the body uneasy to crack and light. The manufacturing effects of different leather can also be very different, for example, because it's thin, we use the sheepskin as the body, can wrap it on the wooden body and sculpture. We would first paint lacquer on the shape and do the mother-of-pearl mosaic, gold silver mosaic, bone-stone mosaic and eggshell mosaic according the decoration demands, or stick on gold or silver foil according to the patterns when it's about to dry. Then we would do mask dyeing, polish and push light, and finally make a unique, gorgeous and elegant bag of lacquer art. In this way, the surface of the bag's body will be glossy and the trace of interlacing will not be clear. Or we can use leathers with clear texture, like cow skin and crocodile skin, etc, and paint some layers of different colors making use of the clear texture, and then polish to make the rich color changeable.

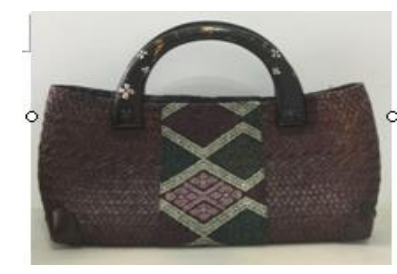

Fig. 2. The application method in the design of the bag of gold 


\section{The Application of Lacquer Decoration Technology in the Bags' Bodies}

The bag's body has the "absolute position" in the bag design. In Pic-5, we would use the sandpaper to polish the bag with a shape of a "box", and paint a layer of primer and throw some silver flour when the primer is about to dry, and paint a layer of clear lacquer and place it in the shade, and then paint red and orange lacquer, and do the mother-of-pearl mosaic when it's half dry. We would continue to paint green, white and black lacquer and use sandpaper to polish slowly when it is completely dry, and at last paint a layer of clear lacquer again. Because of the different polishing power and asymmetric color distribution, we will get a colorful effect.

In Pic-6, the bag is the black cubic fan-shaped chain bag. In order to enrich the decoration effect, we would paint a layer of black lacquer below the fan shape, and decorate it in the way of eggshell mosaic and paint a layer of clear lacquer on the fan shape and throw some gold flour. The distinctive bag is finished when it is completely dry. The gold flour will shine in different luminous conditions, which greatly improve the visual effect of the bag.

\section{The Application of Lacquer Decoration Technology in the Bags of Materials like Cotton and Hemp, and Knitting, etc.}

Different fabrics would be often split in bag designs, for example, designers would inlay and split fabrics like leather, cotton and linen and so on, with each other through the crafting techniques, like sticking, painting, varnishing and pouring, etc, which can not only keep the texture of the fabrics, but also integrate all kinds of decoration materials, like gold, silver, lead, tin, shells, eggshells, stone flakes and wooden flakes, etc, into the inlaying and splitting position, to make the decoration mix reality and fantasies together, contrast strongly and make the decorative effect distinctive.

Cotton and hemp material, and knitting material are used in traditional bags, which is light and convenient to use. If we put the mosaic technology of lacquer decoration technology into the inlaying of the bag's material, use the craft technique like sticking, or drawing, or painting, or showering, we can not only keep the texture of knitting, cotton and hemp, but also integrate all kinds of decoration materials like gold, silver, lead, tin, shell, eggshell, stone slice and wooden slice, etc, into the inlaying part. The decoration, mixing reality and fantasies together and looking splendid in green and gold, can show the perfect matching effect. In Pic-7, we paint a layer of clear lacquer on the wooden knitting bag and paste some silver foil, and then paint again, in this way, we make a bag with a sense of future. Compared to the traditional bag decoration materials, the gloss of the lacquer and the warm feel are incomparable.

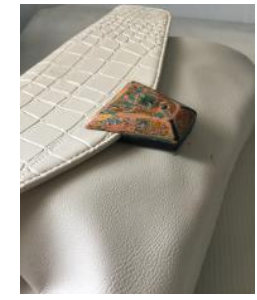

Fig. 3. Lacquerware is used with the buckle of the bag

\section{CONCLUSION}

For women, bags like "spell" are the unforgettable consumer goods. The "magic power" is the practicability of bags, but is more about its satisfying women's dream and secrets, the thoughts with intelligence [1]. Bags need "focuses" to be lively and remarkable. The selective applications of Lacquer decoration technology, a kind of traditional processing craftwork with unique beauty, in bag design, greatly improve the artistry of modern bag design, show unique gloss and feel, also extend the bags' service life and make the modern bags not only be practical in use, but more have art collection value.

\section{ACKNOWLEDGMENT}

T HUA Mei. bags and suitcases, Beijing: China Morden Economics Publishing House, 2008.

\section{REFERENCES}

[1] LI Jiang . Is used, the lifeblood of the lacquer culture . Decoration: 2012;02.

[2] Zhi Zhou ,De-quan Huang ."Device" calmly -- lacquer art professor liang yuan home interview . Decoration: 2012;02.

[3] LI Jiang . Coming from the hemudu - 2006 China international symposium on the development of modern lacquer art .Yangzhou peak BBS: 2006.

[4] LIn-ping $\mathrm{Xu}$.Traditional techniques and modern lacquer art .Literature and art research: 2012;05.

[5] Ji-jun Weng . Lacquer art.Shang hai: Shanghai science and technology education publishing house: 2006.

[6] Juan Xu . Try to talk about China's early Zhu Zhiyun lacquer color ink .Literary and artistic life:2010;04.

[7] Yang Gao. Chinese traditional decoration and modern design .Fujiang: Fujian fine arts publishing house: 2005.

[8] Da-kai Du.Tsinghua university fine arts.Beijing : Tsinghua university press: 2006.

[9] Cheng-zhang Huang. Xiu ACTS the role of record drawings.Shang dong: Shandong pictorial publishing house:2007.

[10] Shi-guang Qiao. Lacquer art.Hang zhou: China academy of fine arts publishing house: 2000 .

[11] Mei Hua. Bags.Beijing:China economic times press:2008.

[12] Fu-yun Zhang, Yu-er Wu. Costume design art.Beijing:Chemical industry press: 2012.

[13] Xue-mei Li. Contemporary bags on the shape and structure of innovative design.Art design research:2009;04.

[14] Juan Lin . Also the lacquer art "in the stereo modelling of" lacquer "-lacquer art creative teaching.Decoration: 2003;08.

[15] Zhi-qiang Gao. Evolution theory of lacquer ware fetal bone.His art: 1997:01. 